\title{
Endothelial Cells Regulate Cardiac Myocyte Reorganisation Through $\beta 1$-Integrin Signalling
}

\author{
Yu Zhang ${ }^{\mathrm{a}, \mathrm{c}}$ Hai Lib,c Renyue Wei ${ }^{\mathrm{a}}$ Jing Ma ${ }^{\mathrm{a}}$ Yanhua Zhao ${ }^{\mathrm{a}}$ Zhengxing Lian ${ }^{\mathrm{c}}$ \\ Zhonghua Liu ${ }^{\mathrm{a}}$ \\ ${ }^{a}$ College of Life Science, Northeast Agricultural University, Harbin, ${ }^{b}$ State Key Laboratory of Veterinary \\ Biotechnology, Harbin Veterinary Research Institute, The Chinese Academy of Agricultural Sciences, \\ Harbin, 'College of Animal Science and Technology, China Agricultural University, Beijing, P.R. China
}

\section{Key Words}

Endothelial cell $•$ Cardiac myocyte $\bullet \beta 1$-integrin $•$ Myocardial infarction

\begin{abstract}
Background: In normal hearts, capillaries are densely distributed throughout the myocardial tissue, and the cross-talk between myocytes and capillary endothelial cells plays a pivotal role in regulating cardiac development, maturation and function. Although previous studies have suggested a role for the endothelium in the organisation of nearby cardiomyocytes, the underlying mechanism has yet to be illustrated. Methods and Results: Using a transwell coculture system, we studied the paracrine effect of endothelial cells on cardiomyocytes and found that the regulation of cardiomyocyte spatial reorganisation and cytoskeletal dynamics by endothelial cells was coupled with $\beta 1$-integrin induction. To determine the role of $\beta 1$-integrin in this process, we preincubated myocytes with a $\beta 1$-integrin function-blocking antibody before coculture. $\beta 1$-integrin blockage abolished myocyte chemotactic activity and inhibited microtubule extension and stress fibre assembly. We further evaluated the therapeutic potential of combined endothelial cell-cardiac myocyte transplantation against ischemic cardiomyopathy in an acute myocardial infarction (AMI) mouse model. The results showed that myocytes and endothelial cells synergistically promoted ischemic myocardial repair, as evidenced by the robust engraftment and migration of implanted cells within the infarcted area, as well as the stimulation of angiogenesis, the attenuation of scar tissue and the improvement of cardiac function. Conclusion: Our study demonstrated the necessity of $\beta 1$-integrin in the interactions between cardiomyocytes and endothelial cells and presented a novel combined transplantation approach that might hold promise for treating ischemic cardiomyopathy.
\end{abstract}

Y. Zhang and $\mathrm{H}$. Li contributed equally to this work.

Zhengxing Lian,

and Zhonghua Liu,

KARGER 125
College of Animal Science and Technology, China Agricultural University, Beijing 100193 (China) and College of Life Science, Northeast Agricultural University, Harbin 150030 (China); E-Mail lianzx@cau.edu.cn, E-Mail liu086@yahoo.com 


\section{Introduction}

Organ morphogenesis is an intricate process in which cells derived from different sources interact, coalesce and ultimately develop into a mature organ [1]. This process is rigidly regulated by cell-cell interactions to ensure that the mature organ obtains proper size, structure and function.

Within a normal heart, capillaries are located next to almost every cardiac myocyte [2], and the cross-talk between myocytes and capillary endothelial cells is involved in cardiac development, maturation and function. Previous results from developmental biology showed that myocardial cell migration and maturation depend on the presence of endocardial endothelium at early stages [3]. At later stages, myocardial capillaries not only modulate cardiac performance and rhythmicity by producing nitric oxide and endothelin [4], but also maintain cardiac function under certain stress conditions [5]. Experiments in which VEGF was inactivated or overexpressed showed that both excessive and deficient microvessel formation resulted in cardiac dysfunction [3]. In addition, some recent studies demonstrated that myocytes also contributed to endothelial cell proliferation and angiogenesis [6, 7], which could be attenuated by competitively blocking VEGF signalling, indicating the role of myocyte-derived VEGF in paracrine regulation of endothelial cells $[8,9]$. Although more and more findings highlight the significance of cross-talk between cardiac myocytes and the endothelium in normal cardiac function, few studies have focused on the mechanisms underlying these interactions.

Since the introduction of cell transplantation as a promising strategy for restoring cardiac function after myocardial injury, researchers have investigated a variety of cell sources for cardiac repair. Because endothelial cells have the potential to fuse into capillary networks both in vivo and in vitro, intramyocardial transplantations of endothelial cells or their progenitors have been conducted in myocardial ischemic animal models for therapeutic evaluation [10,11]. As expected, local or systemic administration of endothelial progenitor cells enhanced ischemic neovascularisation and cardiac function to varying degrees [10, 12]; however, the underlying mechanism is still ambiguous. In addition, there is a growing recognition that the majority of implanted cells will die shortly after transplantation [13], suggesting that additional support is needed for their survival.

In the present study, we characterised the effect of endothelial cells on cardiac myocyte reorganisation and cytoskeletal assembly using a membrane insert coculture system, evaluated the therapeutic potential of combined endothelial cell-cardiac myocyte transplantation in an acute myocardial infarction (AMI) mouse model and demonstrated the essential role of $\beta 1$-integrin signalling in the cross-talk between these two cell populations.

\section{Material and Methods}

\section{Cell isolation and identification}

All animal experimental procedures and protocols in this study were approved by the China Agricultural University Animal Care and Use Committee.

Mouse cardiac myocytes (CMs) were obtained from neonatal C57BL/6 mice as described previously [3]. Briefly, hearts were isolated, minced and incubated with $0.05 \%(\mathrm{w} / \mathrm{v})$ trypsin overnight at $4^{\circ} \mathrm{C}$, then digested at $37^{\circ} \mathrm{C}$ with $0.1 \%(\mathrm{w} / \mathrm{v})$ collagenase type-II. The released cells were filtered through a 200 mesh stainless-steel filter to obtain homogeneous cells and preplated for $2 \mathrm{~h}$ to remove attached cardiac fibroblasts. CMs were maintained in DMEM supplemented with 10\% FCS. Mouse endothelial cells (ECs) were isolated from the lungs of neonatal C57BL/6 mice as described previously [14] and cultured on matrigel-coated plates in DMEM supplemented with 10\% FCS, $150 \mu \mathrm{g} / \mathrm{L}$ VEGF and $100 \mu \mathrm{g} / \mathrm{L}$ heparin.

EC surface markers were identified by immunocytochemistry using antibodies against von Willebrand factor and CD31. To further confirm EC function, the uptake of Dil-labelled acetylated low density lipoprotein (Dil-AcLDL, Invitrogen) was measured according to the manufacturer's protocol. Briefly, $1 \mathrm{mg} / \mathrm{ml} \mathrm{Dil-}$ AcLDL was added to the cultures and incubated for $3-4 \mathrm{~h}$ at $37^{\circ} \mathrm{C}$. The cells were then washed with PBS, and the uptake of DiI-AcLDL was detected by fluorescence microscopy. For CM identification, an anti-desmin 


\section{Cellular Physiology Cell Physiol Biochem 2015;35:1808-1820

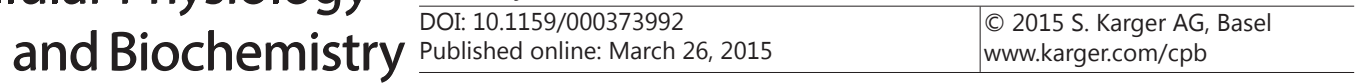

Zhang et al.: $\beta 1$-Integrin Mediated Endothelium-Cardiomyocyte Interaction

antibody was used to identify muscle cells, and an antibody against smooth muscle-specific $\alpha$-actin was used to exclude vascular smooth muscle cells. Cells between passage 2-5, and with more than $95 \%$ purity, were used in the following experiments.

\section{Cell culture}

For mixed-population cultures, ECs and CMs were seeded at the same time at a ratio of 1:2 on a 6-well plate coated with matrigel; the seeding density was $7.5 \times 10^{4}$ cells $/ \mathrm{cm}^{2}$. For the Control, CMs were seeded alone at a density of $5 \times 10^{4}$ cells $/ \mathrm{cm}^{2}$.

A transwell filter with an 8- $\mu$ m pore size PET membrane (Falcon, Becton Dickenson Labware) was used for the chemotaxis experiments, and ECs and CMs were cultured separately on opposite sides of the filter. Briefly, ECs were seeded at a density of $5 \times 10^{4} \mathrm{cells} / \mathrm{cm}^{2}$ on a $0.1 \%$ gelatin-coated filter and proliferated in DMEM supplemented with $10 \%$ FCS for $24 \mathrm{~h}$, at which point they reached $60 \%$ confluency. The filter was then placed EC-side down into a 6-well plate, and CMs were seeded on the outer side of the filter at a density of $5 \times 10^{4}$ cells $/ \mathrm{cm}^{2}$. For the Control, CMs were plated on the outer side without prior seeding of the ECs on the inner side. To study $\beta 1$-integrin function, CMs were preincubated for $1 \mathrm{~h}$ with either $10 \mu \mathrm{g} / \mathrm{ml}$ of function-blocking monoclonal antibody directed against mouse $\beta 1$-integrin (BD Biosciences) or $10 \mu \mathrm{g} /$ $\mathrm{ml}$ IgG Armenian hamster monoclonal antibody as an isotype control, after which the cells were seeded as described above.

\section{Immunocytochemistry}

For immunocytochemistry analysis, cultures were washed with PBS and fixed in 3.7\% formaldehyde for $10 \mathrm{~min}$ at room temperature. After excess formaldehyde was quenched with PBS, the cells were permeabilised with $0.1 \%$ Triton-X100 for 30 min. Nonspecific antibody binding was blocked with $2 \%$ bovine serum albumin for $1 \mathrm{~h}$, and the samples were then incubated with primary antibodies overnight at $4^{\circ} \mathrm{C}$, followed by incubation with the appropriate secondary antibodies for 30-60 min at room temperature. Cell nuclei were stained with Hoechst 33342 (Sigma Aldrich). The samples were observed using an Olympus IX81 inverted microscope (Olympus Optical), and U-MWIBA (FITC), U-MWIB (TRITC/Cy3/Rhodamine) and U-MWU (Hoechst 33342) filter sets were used to observe the fluorescence. The following primary antibodies were used: Rat anti-CD31 monoclonal antibody (clone MEC7.46, Abcam), Sheep anti-von Willebrand factor polyclonal antibody (Abcam), Rat anti- $\beta 1$-integrin monoclonal antibody (clone MB1.2, Merck Millipore), Rabbit anti-desmin monoclonal antibody (clone Y66, Merck Millipore), Rabbit anti-alpha smooth muscle actin polyclonal antibody (Abcam), FITC-labelled anti- $\beta$-tubulin monoclonal antibody (clone Tub2.1, Abcam) and TRITC-labelled anti-paxillin monoclonal antibody (clone 349/Paxillin, BD Transduction Laboratories). Rhodamine-phalloidin (Sigma), which can specifically recognise filamentous actin, was also used to label microfilament cytoskeleton.

Real-time qPCR

Total RNA was isolated from cells or tissues using Trizol (Invitrogen) and reverse transcribed using the SuperScript III First-Strand synthesis kit (Invitrogen) according to the manufacturer's protocol. RT-qPCR was performed using the SYBR PrimeScriptTM Kit (TaKaRa) on an ABI 7500 Real-Time PCR System (Life Technologies) with $\beta 1$-integrin primers (sense: 5'-GCCAGGGCTGGTTATACAGA-3'; antisense: 5'-TCACAATGGCACACAGGTTT-3').

\section{Apoptosis analysis}

To detect cell apoptosis, chromatin was labelled with Hoechst 33342, and morphological observation was performed using fluorescence microscopy. Annexin V/PI assays were carried out using the Annexin V-FITC/PI apoptosis detection kit (Nanjing Keygen Biotech) as described previously [15], and both adherent and floating cells were collected for flow cytometric analysis (FACSCalibur BD Biosciences). The data were analysed using ModFit Cell Cycle Analysis Software (Verity Software House). For each sample, $1 \times 10^{4}$ events were recorded.

Acute myocardial infarction (AMI) mouse model and cell transplantation

C57BL/ 6 female mice at 10-12 weeks of age were used as recipients. MI was induced by ligation of the left anterior descending coronary artery (LAD) as described previously [13]. Briefly, mice were anesthetised 


\section{Cellular Physiology Cell Physiol Biochem 2015;35:1808-1820

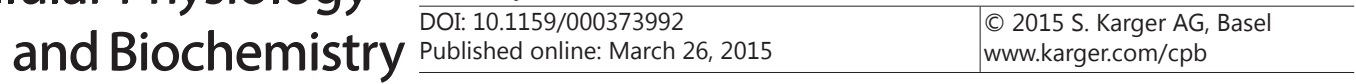

intraperitoneally with $60 \mathrm{mg} / \mathrm{kg}$ pentobarbital sodium followed by $10 \mathrm{mg} / \mathrm{kg}$ ketamine, and oxygen (2 L/ min) was provided by an oxygen delivery system. A small hole was made in the fourth intercostal space using a mosquito clamp, and the heart was exposed and gently popped out. The LAD was ligated between the left atrium and the right pulmonary outflow tract using a 7/0 suture. The correct position of the ligation was assessed by the colour change of the ischemic area.

At $7 \mathrm{~d}$ after AMI, the mice received a second thoracotomy for cell transplantation. They were randomly divided into four groups for the different treatments: Control group (10 $\mu \mathrm{l} \mathrm{PBS,} \mathrm{n=12),} \mathrm{CM} \mathrm{group} \mathrm{(10} \mu$ l PBS containing $\left.10^{6} \mathrm{CMs}, \mathrm{n}=12\right)$, EC group (10 $\mu$ PBS containing $\left.10^{6} \mathrm{ECs}, \mathrm{n}=12\right)$ and CM+EC group $(10 \mu \mathrm{PBS}$ containing $5 \times 10^{5} \mathrm{CMs}$ and $5 \times 10^{5} \mathrm{ECs}, \mathrm{n}=12$ ). The areas for intracardiac injection were visually selected by pale colour and wall motion akinesis, and cells were transplanted into marginal zones of MI using a 32-gauge needle (Hamilton, USA) at three distinct but adjacent sites. All transplanted cells were labelled with PKH67 (Sigma-Aldrich) according to the manufacturer's instructions. Cyclosporine A (Neoral) was orally administered daily at a dose of $20 \mathrm{mg} / \mathrm{kg}$, beginning on the day of transplantation and continuing postoperatively.

\section{Histological analysis}

Animals were sacrificed on day 10 or day 28 post transplantation. The hearts were harvested, and the ischemic tissues were isolated, embedded in OCT (Tissue Tek, Inc.) and snap-frozen in liquid nitrogen. Then, 10- $\mu$ m sections were made using a freezing microtome (Leica, Nussloch, Germany) and examined by fluorescence microscopy to evaluate implanted cells labelled with PKH67. The engrafted cell fraction was calculated as the green cell area/myocardium area ratio in a random field using Image-Pro software (Image-Pro for Windows, Media Cybernetics). Frozen sections were stained with hematoxylin and eosin for morphological observation. The scar area fraction was calculated as the scar area/myocardium area ratio in a random field after Masson's trichrome staining. An anti-CD31 antibody was used to identify microvessels in the infarct regions, and immunostaining was performed according to Bilousvoa et al. [16]. The CD31+ circular structures were counted in a random field, and microvessel density was expressed as the number of CD31+ structures $/ \mathrm{mm}^{2}$.

For each histological feature, namely, microvessel density, scar area fraction or engrafted cell fraction, 5-7 random fields were observed. Each field was examined by two independent observers and then averaged. The averages were used for statistical analysis.

\section{T-lymphocyte subset analysis}

Mice were sacrificed $10 \mathrm{~d}$ after transplantation, and their spleen mononuclear cells were isolated by density gradient centrifugation [17]. The proportions of $\mathrm{CD}^{+}$and $\mathrm{CD}^{+} \mathrm{T}$ cells were assayed by FACS following our previous publication [18], and the ratio of $\mathrm{CD}^{+} / \mathrm{CD}^{+} \mathrm{T}$ cells was calculated. The FITC-labelled anti-CD4 and FITC-labelled anti-CD8 monoclonal antibodies used in the study were purchased from Santa Cruz Biotechnology, Inc.

\section{Echocardiogram}

Cardiac function was estimated on day 28 after transplantation using a cardiovascular ultrasound system (GE Healthcare). The mice were anesthetised with ketamine $(10 \mathrm{mg} / \mathrm{kg}$ ) and placed on a warm blanket. Two-dimensional guided M-mode echocardiograms were obtained using a 10-MHz linear-array transducer. The LV end-diastolic diameter (LVIDd), LV end-systolic diameter (LVIDs), interventricular septum thickness at end diastole (IVSd) and LV posterior wall thickness at end diastole (PWd) were measured. Fractional shortening (FS) was calculated as FS(\%)=[(LVIDd-LVIDs)/LVIDd] $\times 100$.

\section{Statistical analysis}

Data were presented as the mean \pm standard deviation. Data analysis was performed with SPSS software package (SPSS for Windows, Release 10.0.1, SPSS Inc., Chicago, IL, USA) using unpaired student's t-test or one-way ANOVA followed by Newman-Keuls test. Differences with $p<0.05$ were considered statistically significant. 


\section{Results}

\section{Endothelial cells regulate cardiac myocyte reorganisation}

Isolated ECs were identified by the presence of the endothelial markers CD31 and von Willebrand factor via immunofluorescence staining (Fig. 1A-B). In addition, the ECs took up Ac-LDL and exhibited tube-like structures on matrigel (Fig. 1C-D). Statistical results showed that $92.83 \pm 3.06 \%$ of the isolated CMs were positive for desmin, a marker of muscle cells (Fig. 1E), but few of them expressed $\alpha$-smooth muscle actin, a marker of vascular smooth muscle cells (VSMCs) (Fig. 1F), thereby excluding probable infiltration by VSMCs.

CMs were cultured alone or cocultured with ECs to investigate the interactions between CMs and ECs. After $2 \mathrm{~d}$ of cultivation on matrigel, we observed capillary-like networks in the CMs-ECs coculture group (Fig. 2B-C), while similar structures were not found in the CMs alone group (Fig. 2A), indicating an indispensable role of ECs in the formation of networklike structures. Immunofluorescence staining followed with statistical analysis showed that $96.83 \pm 2.40 \%$ of ECs and $89.50 \pm 4.37 \%$ of CMs were located within these networks, and after $7 \mathrm{~d}$ of coculture, ECs formed tube-like structures with CMs spread along the outer sides (Fig. 2D), suggesting that CMs preferred migrating towards and staying beside ECs. Given the vasculogenic potential of ECs, these results indicated a spatial reorganisation of CMs that is driven by ECs.

\section{$\beta 1$-integrin signalling is required for the migration of CMs toward ECs}

To determine whether the reorientation of CMs by ECs requires cell-cell contact, the migration of CMs was examined in a transwell filter culture system, with ECs and CMs seeded separately on opposite sides of the filter. After $24 \mathrm{~h}$ of cultivation, the transmigration of CMs from the upper side to the lower side was evaluated by immunofluorescence staining for desmin. As shown in Fig. 3B, a number of desmin-positive cells were present on the lower side, which was seeded with ECs, accounting for $20.50 \pm 1.87 \%$ of the total cells, but few desmin-positive cells were observed in the control group (without ECs on the lower side, Fig. 3A), suggesting that the EC-induced chemotactic activity of CMs is contact independent.

To investigate the effect of ECs on the expression of $\beta 1$-integrin by CMs, immunofluorescence staining was performed on CMs maintained on the upper side after 24 h of cultivation. As shown in Fig. 3E-F, CMs cocultured with ECs expressed higher levels of $\beta 1$-integrin compared with the control group, especially at focal adhesions (arrow). In the meantime, a similar result was also obtained at transcriptional level by RT-qPCR (Fig. 3G), suggesting a stimulatory effect of ECs on $\beta 1$-integrin expression in CMs.

To determine the role of $\beta 1$-integrin in the EC-induced chemotaxis of CMs, we preincubated CMs with a $\beta 1$-integrin function-blocking antibody before transwell coculture. The results showed that the migratory ability of CMs toward ECs was seriously impaired by blocking $\beta 1$-integrin compared with the isotype IgG control group (Fig. 3C-D, $2.17 \pm 1.17 \%$ vs. $18.33 \pm 3.14 \%, p<0.05)$. Furthermore, the inhibitory effect was dose dependent, reaching a plateau at $10 \mu \mathrm{g} / \mathrm{ml}$ (Fig. $3 \mathrm{H}$ ). Given the pivotal role of $\beta 1$-integrin in myoblast survival [15], we investigated the effect of $\beta 1$-integrin blockage on apoptosis in CMs. The results showed that apoptosis was not induced by the $\beta 1$-integrin function-blocking antibody at our experimental dose, as evidenced by chromatin morphology (Fig. 3I-J) and apoptotic population analysis (Fig. 3K-L), suggesting that the reduced chemotactic activity of CMs towards ECs was not due to cell apoptosis.

\section{ECs promote a $\beta 1$-integrin-dependent cytoskeletal reorganisation in CMs}

At the beginning of $\mathrm{CM}$ transmigration, the morphologies of CMs were compared among the CMs-alone group (Fig. 4A), the CM-EC cocultured group (Fig. 4F) and the CM-EC cocultured group preincubated with $\beta 1$-integrin function-blocking antibody (Fig. 4K). The results showed that more CMs spread after $4 \mathrm{~h}$ of coculture with ECs, which was hindered by $\beta 1$-integrin blockage.

Given that cytoskeletal reorganisation plays an important role in cell migration, we investigated microtubule and microfilament dynamics in CMs at the beginning of CM 
Fig. 1. Identification of isolated ECs and CMs. (A-B) Putative ECs were labelled with antibodies targeting CD31 (A) or von Willebrand factor (B) followed by FITC-conjugated secondary antibody. Cell nuclei were stained with Hoechst 33342. (C) Putative ECs showed uptake of Dil-AcLDL. (D) Putative ECs formed tube-like structures on matrigel with delayed medium exchange. (E-F) Putative CMs were labelled with antibodies targeting desmin (E) or $\alpha$-smooth muscle actin (F) followed by FITC-conjugated secondary antibody. Nuclei were stained with Hoechst 33342. Scale bars $=100 \mu \mathrm{m}$.
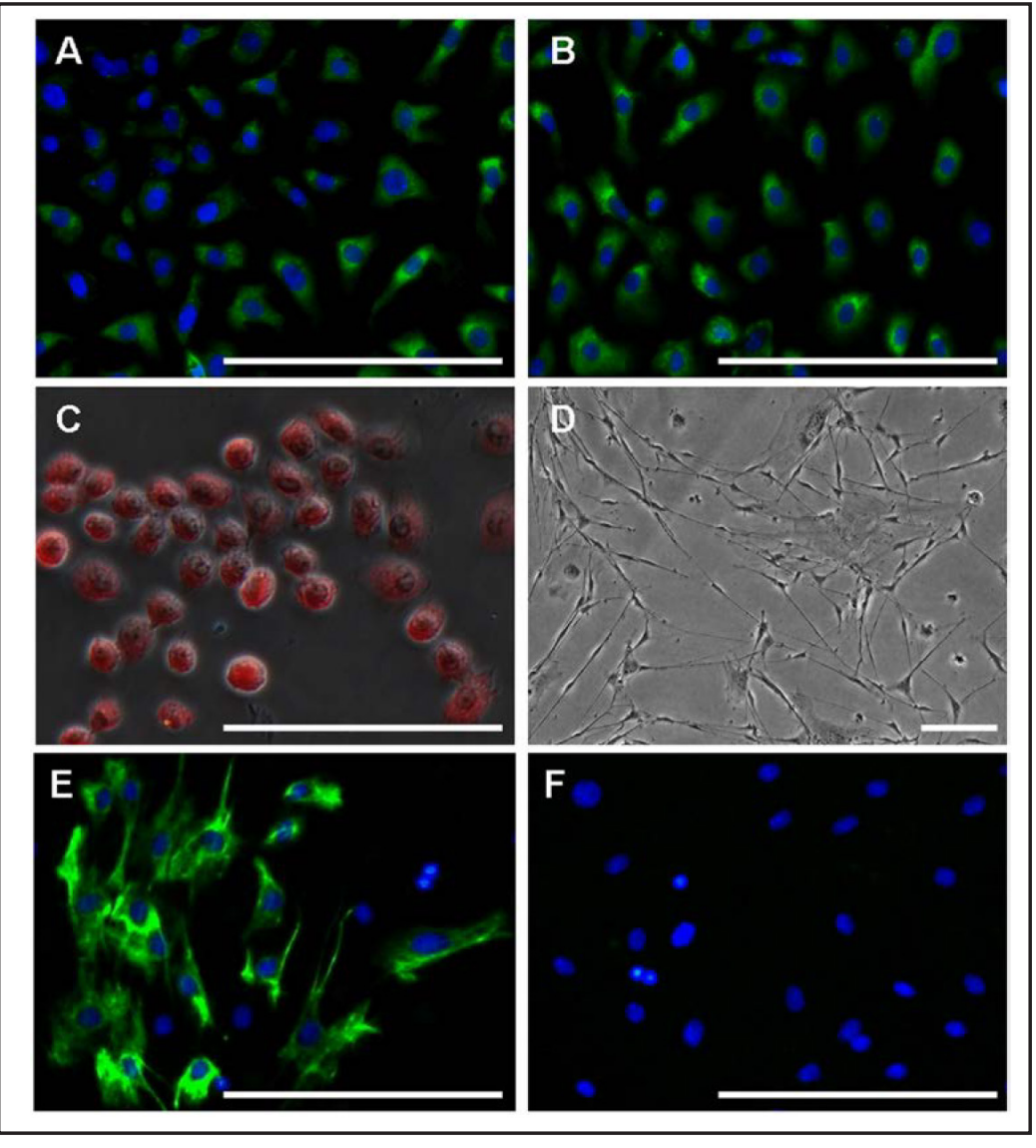

Fig. 2. ECs promote $\mathrm{CM}$ reorganisation. (A) $\mathrm{CMs}$ were cultured alone for 2 d. (B-D) CMs were cocultured with ECs for 2 (B-C) or $7 \mathrm{~d}$ (D). (C-D) Double immunofluorescence staining of structures in EC-CM cocultures at day 2 (C) or day 7 (D). ECs were labelled with Cy3-conjugated CD31 antibodies (Red). CMs were labelled with FITC-conjugated desmin antibodies (Green). Cell nuclei were visualised with Hoechst 33342 (Blue). Scale bar $=100 \mu \mathrm{m}$.
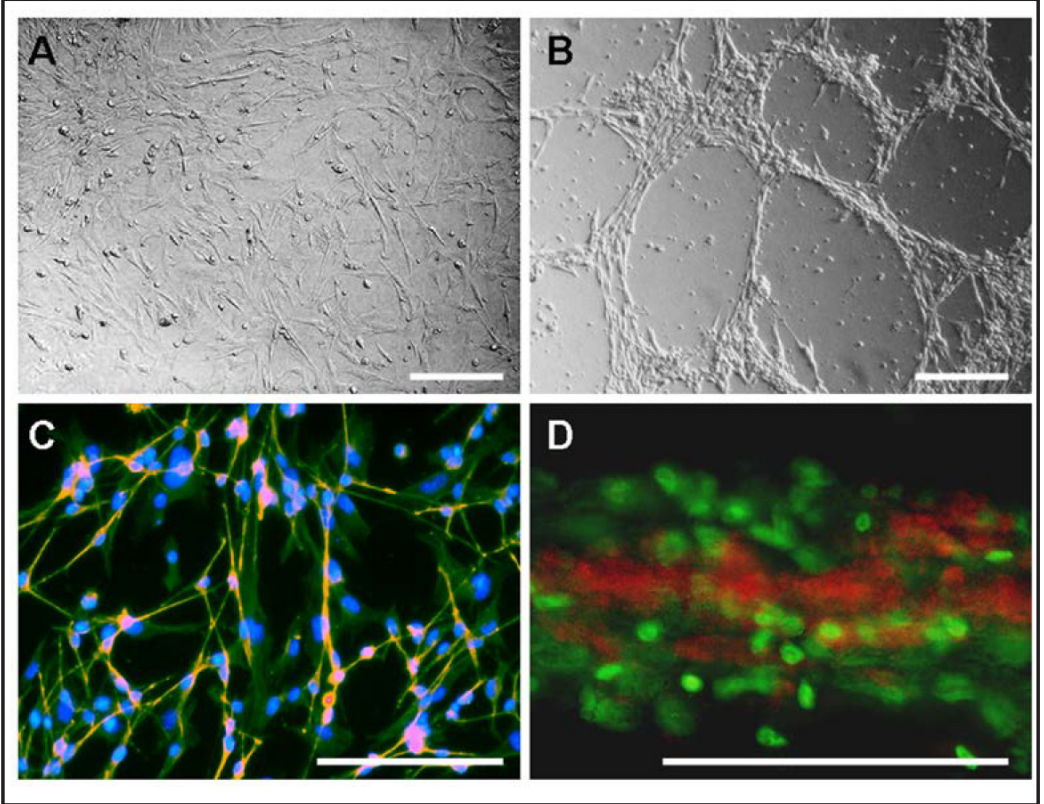

transmigration. After $4 \mathrm{~h}$ of cultivation, CMs cocultured with ECs formed an integrated filamentous F-actin cytoskeleton parallel to their migratory direction (Fig. 4J), whereas actin filaments in CMs cultured alone appeared diffuse and less organised (Fig. 4E). At the same time, paxillin distributed in a dotted pattern at the periphery of cocultured CMs (Fig. 4J, inset), indicating that typical focal adhesions were formed; however, we did not observe a similar paxillin distribution in CMs cultured alone until $7 \mathrm{~h}$ of cultivation. Migrating cells are 


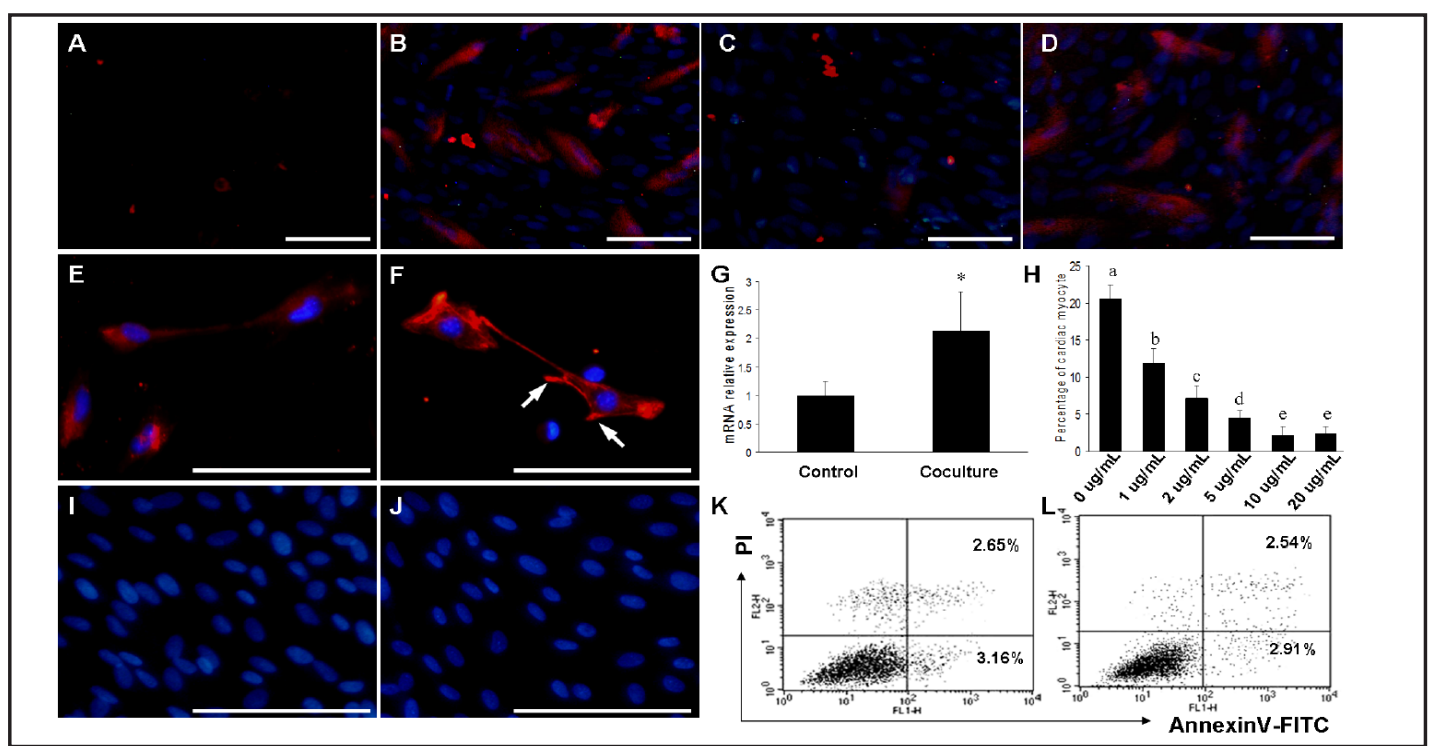

Fig. 3. $\beta 1$-integrin is required for the chemotactic activity of CMs toward ECs. (A-D) After 24 h of cultivation, the transmigration of CMs was assayed in the CMs-alone group (A), the CM-EC cocultured group (B), the CM-EC cocultured group preincubated with $\beta 1$-integrin function-blocking antibody (C) and the CM-EC cocultured group preincubated with isotype IgG antibody (D) by immunofluorescence staining using anti-desmin antibodies. (E-G) CMs were cultured alone (E) or cocultured with ECs (F) for $24 \mathrm{~h}$, and $\beta 1$-integrin expression levels were detected by immunofluorescent staining using anti- $\beta 1$-integrin antibodies or RT-qPCR (G). $n=4$. Data are means \pm standard deviation. ${ }^{*} p<0.05$. $(\mathrm{H})$ Transmigration of CMs was assayed in the CM-EC cocultured group preincubated with the indicated concentrations of a $\beta 1$-integrin function-blocking antibody. $n=6$. Data are means \pm standard deviation, and values with different superscripts indicate significant differences $(p<0.05)$. (I-L) CMs without (I, K) or with (J, L) $10 \mu \mathrm{g} / \mathrm{ml} \beta 1$-integrin blocking antibody treatment were cocultured with ECs for $24 \mathrm{~h}$ and then subjected to Hoechst33342 staining to observe chromatin morphology (I, J) or FITC-Annexin V and PI costaining to analyse the apoptotic population (K, L). Scale bar $=50 \mu \mathrm{m}$.

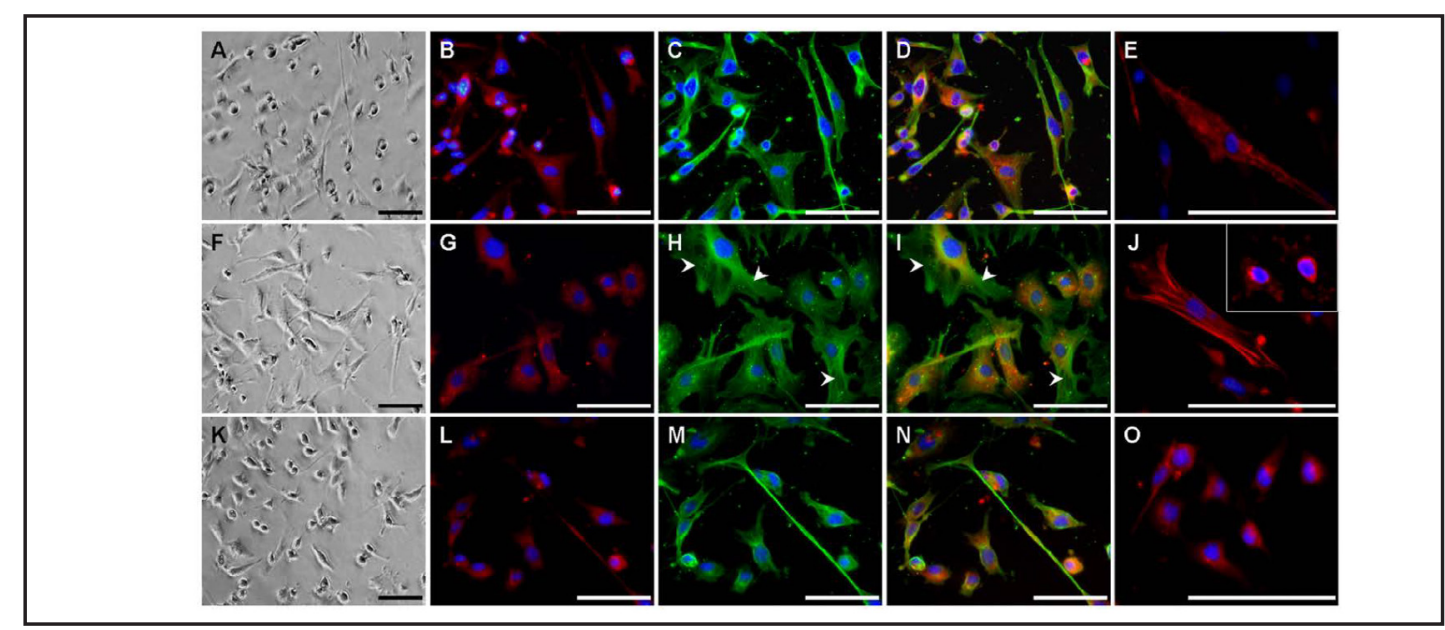

Fig. 4. ECs promote CM cytoskeletal assembly in a $\beta 1$-integrin-dependent manner. After $4 \mathrm{~h}$ of cultivation, the cytoskeletal dynamics of CMs were investigated in the CMs-alone group (A-E), the CM-EC cocultured group (F-J) and the CM-EC cocultured group preincubated with $\beta 1$-integrin function-blocking antibody (K0). (A, F, K) Bright field. (B, G, L) CMs were labelled with Cy3-conjugated desmin antibodies (Red). (C, H, M) CMs were labelled with FITC-labelled $\beta$-tubulin antibodies (Green). (D, I, N) Merged images of the desmin and $\beta$-tubulin staining. (E, J, O) Actin filaments and focal adhesions were visualised by staining with Rhodamine-phalloidin and TRITC-labelled paxillin antibodies (J, inset), respectively. Cell nuclei were stained with Hoechst 33342 (Blue). Scale bar $=50 \mu \mathrm{m}$. 


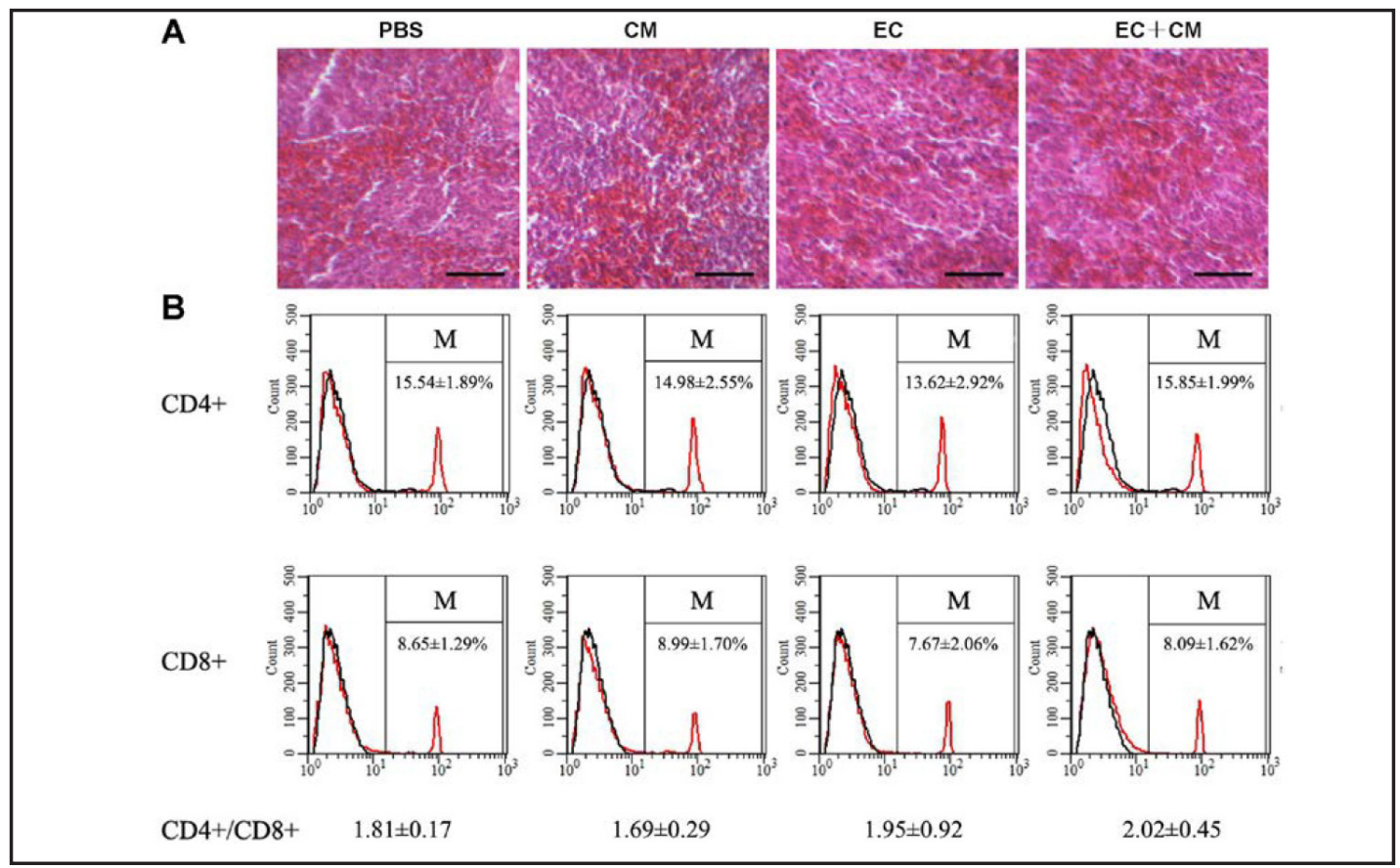

Fig. 5. Evaluation of acute rejection response in the transplanted groups. (A) At $10 \mathrm{~d}$ after transplantation, mice from the indicated groups were sacrificed, and the spleens were isolated and sectioned for hematoxylin and eosin staining. Scale bar $=100 \mu \mathrm{m}$. (B) The proportions of spleen CD4 ${ }^{+}$and $\mathrm{CD}^{+} \mathrm{T}$ cells were counted by FACS using FITC-labelled anti-CD4 and FITC-labelled anti-CD8 monoclonal antibodies, and the ratios of $\mathrm{CD}^{+} / \mathrm{CD}^{+}$T cells were calculated. $n=5$ to 7 . Data are means \pm standard deviation.

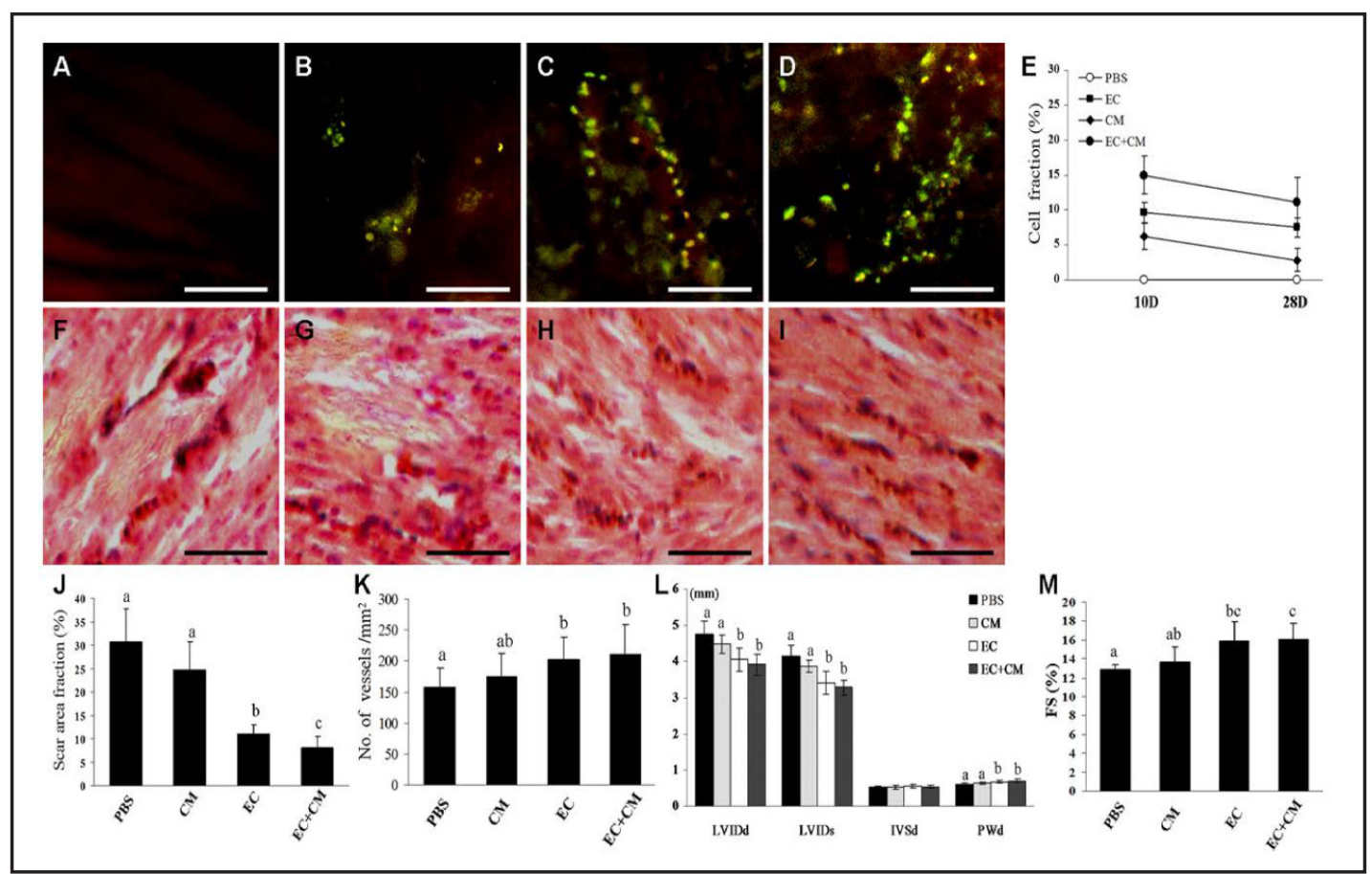

Fig. 6. Combined CM-EC transplantation promotes myocardium organisation and cardiac function. (A-D) At $28 \mathrm{~d}$ after transplantation, cell engraftment was assessed by examining the PKH67-labeled fluorescent cells in control group (A), the CM group (B), the EC group (C) and the EC+CM group (D). (E) The engrafted cell fraction was calculated on day 10 and day 28 after transplantation. $n=5$ to 7 . Data are means \pm standard deviation. (F-I) At 28 days after transplantation, representative images were taken of sections stained with KARGER 


\section{Cellular Physiology Cell Physiol Biochem 2015;35:1808-1820 \begin{tabular}{l|l} 
and Biochemistry Published onIIne: VIarch 26, 2015 & $\begin{array}{l}\text { C 2015 S. Karger AG, Basel } \\
\text { www.karger.com/cpb }\end{array}$ \\
\hline
\end{tabular} \\ Zhang et al.: $\beta 1$-Integrin Mediated Endothelium-Cardiomyocyte Interaction}

hematoxylin and eosin in the control group (F), the CM group (G), the EC group (H) and the EC+CM group (I). (J-K) Scar area fraction (J) and microvessel density (K) were calculated on the 28th day after transplantation. $n=5$ to 7 . Data are means \pm standard deviation, and values with different superscripts indicated significant differences $(p<0.05)$. (L-M) LV end-diastolic diameter (LVIDd), LV end-systolic diameter (LVIDs), end-systolic interventricular thickness (IVSd), LV posterior wall thickness (PWd) (L) and fractional shortening (FS) (M) were measured using a two-dimensional guided M-mode echocardiogram on the 28th day after transplantation. $n=5$ to 7 . Data are means \pm standard deviation, and values with different superscripts indicated significant differences $(p<0.05)$.
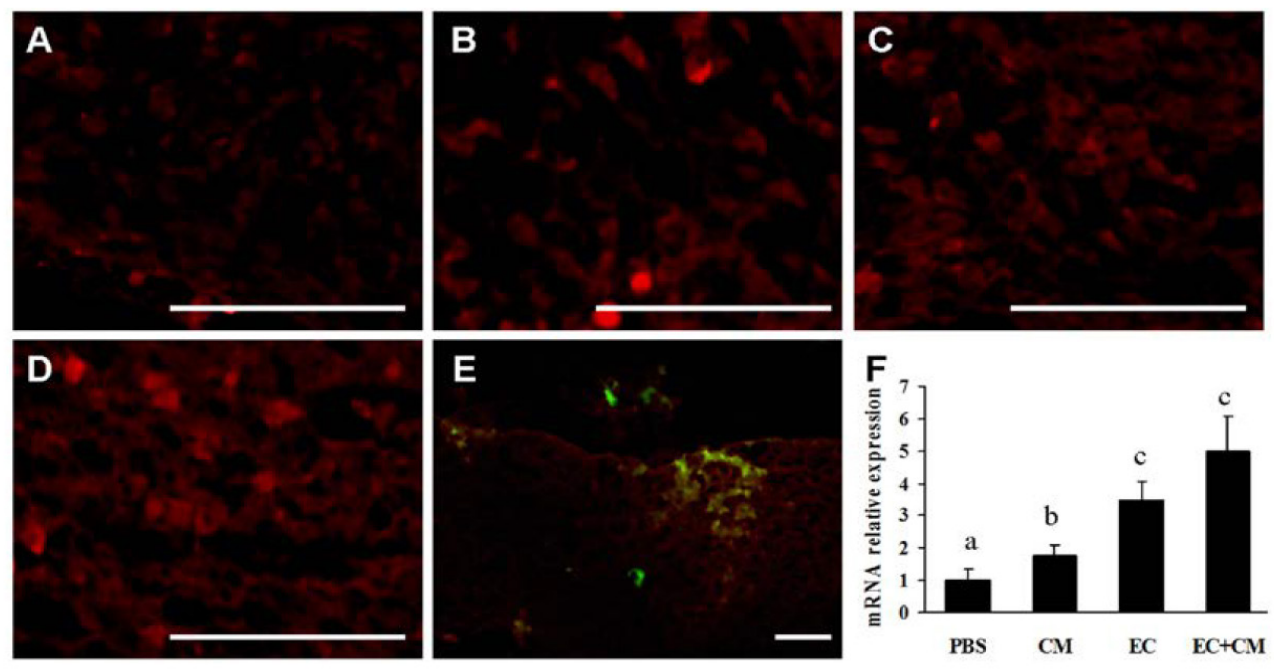

Fig. 7. Combined CM-EC transplantation enhances $\beta 1$-integrin expression levels in the infarcted heart. (AD) Representative images were taken from sections stained with Cy3-conjugated $\beta 1$-integrin antibodies on day 28 after injection with PBS (A), CMs (B), ECs (C) or ECs+CMs (D). (E) At 28 days after transplantation, PKH67-labeled injected cells (Green) were stained with Cy3-conjugated $\beta 1$-integrin antibodies (Red) in the EC+CM group. (F) The transcriptional level of $\beta 1$-integrin was detected by RT-qPCR. $n=4$. Data are means \pm standard deviation, and values with different superscripts indicated significant differences $(p<0.05)$. Scale bar $=50 \mu \mathrm{m}$.

polarised, with a protrusive lamellipodia at the leading edge, followed by the main cell body and a retractable tail. Although lamellipodia formation depends on actin polymerisation, microtubules are essential for directed cell migration and tail retraction [19]. At $4 \mathrm{~h}$ after cultivation, CMs were costained with a Cy3-conjugated desmin antibody and a FITCconjugated $\beta$-tubulin antibody. As shown in Fig. 4G-I, lamellipodia formed by microtubule extension (arrowhead) were observed in most cocultured CMs but only rarely in CMs cultured alone (Fig. 4B-D). These results indicated that ECs promoted stress fibre assembly and microtubule cytoskeleton formation in the cocultured CMs.

Next, we studied the role of $\beta 1$-integrin in this process by incubating CMs with a $\beta 1$ integrin function-blocking antibody before coculture. As shown in Fig. 4L-O, coupled with $\beta 1$-integrin blockage, the promoting effect of ECs on CM microtubule extension and stress fibre assembly were seriously compromised and even worse than those in control group. Besides, the size and intensity of paxillin spots at CM protrusions were greatly reduced with paxillin staining distributing dispersedly within the cytoplasm (data not shown). These results indicated that ECs promoted CM cytoskeleton reorganisation through a $\beta 1$-integrindependent mechanism.

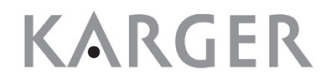




\section{Cellular Physiology Cell Physiol Biochem 2015;35:1808-1820

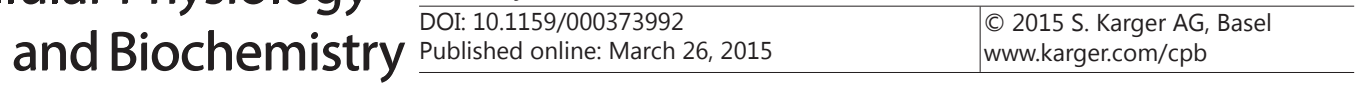 \\ Zhang et al.: $\beta 1$-Integrin Mediated Endothelium-Cardiomyocyte Interaction}

Combined CM-EC transplantation promotes myocardium organisation and cardiac function following AMI

Considering the significance of the interactions between CMs and endothelium in normal cardiac function and the regulatory effects of ECs on the reorientation and cytoskeletal assembly of CMs, the therapeutic potential of CM, EC and combined CM-EC transplantation on ischemic cardiomyopathy were evaluated in a mouse model of AMI. During the experimental period, acute transplant rejection was prevented by using C57BL/6 inbred mice and the immunosuppressant cyclosporine A. As shown in Fig. 5, host antiallograft immune responses were not induced in any group based on spleen histological examination, in which no obvious hyperplasia of follicles was observed (Fig. 5A), and FACS analysis of $\mathrm{T}$ cell subpopulations, wherein the proportions of $\mathrm{CD}^{+}$and $\mathrm{CD} 8^{+} \mathrm{T}$ cells, as well as the $\mathrm{CD}^{+} / \mathrm{CD}^{+}$ratio, were all approximately normal (Fig. 5B).

Cell engraftment was assessed by examining the fluorescence of the PKH67-labeled cells on day 10 and day 28 after transplantation. Compared with other treatments, hearts injected with ECs+CMs contained more PKH67-labeled cells (Fig. 6D-E), and the majority of the cells were engrafted along the host myocardial fibres within the ischemic border zone (Fig. 6D), much like those in the ECs group (Fig. 6C). However, the PKH67-labeled cells in CMs group were less organised and appeared as cell clots (Fig. 6B).

Histological examinations showed that neonatal myocardia were present from the border to the centre of the ischemic area in hearts transplanted with ECs and ECs+CMs (Fig. $6 \mathrm{H}-\mathrm{I})$, although the neonatal myocardia in the ECs group were less organised compared with those in the EC+CM group (Fig. 6H-I). By contrast, massive necrosis and scarring were observed in the CM and control groups (Fig. 6F-G). Statistical analyses revealed that both EC and EC+CM transplantation greatly reduced scar tissue formation within the infarcted area, with reductions of $64.03 \%$ and $72.83 \%$, respectively, compared with the control group (Fig. 6J). Moreover, the EC+CM treatment is more effective than merely injecting ECs alone (Fig. 6J). Microvessel density was determined by immunofluorescence staining for CD31positive structures. Compared with the control and CM groups, capillary density in the infarcted myocardia was increased $28.01 \%$ by EC transplantation and $33.62 \%$ by EC+CM transplantation (Fig. 6K).

To evaluate cardiac function, 2D echocardiograms were performed on the 28th day after transplantation. Left ventricular systolic function, indicated by FS, was significantly improved by EC and EC+CM injection, with elevations of $25.51 \%$ and $24.02 \%$, respectively, compared with the control group (Fig. 6M), along with decreased LVIDd, LVIDs and elevated PWd (Fig. 6L). On the other hand, injecting CMs alone did not improve cardiac function (Fig. $6 \mathrm{~L}-\mathrm{M}$ ), indicating an essential role of ECs in the recovery of cardiac function upon combined transplantation of CMs and ECs.

Combined CM-EC transplantation improves $\beta 1$-integrin expression within the ischemic area

Compared with the other three groups, higher levels of $\beta 1$-integrin were observed within the infarcted areas of hearts injected with ECs+CMs based on analyses of the fluorescent density of Cy3-conjugated antibody targeting $\beta 1$-integrin (Fig. 7A-D). Notably, the region of elevated $\beta 1$-integrin expression tended to colocalise with the PKH67-labeled injected cells in the $\mathrm{EC}+\mathrm{CM}$ group (Fig. 7E), indicating a direct stimulatory effect of $\mathrm{EC}+\mathrm{CM}$ transplantation on $\beta 1$-integrin. A similar pattern of $\beta 1$-integrin expression was also observed at the transcriptional level by RT-qPCR, although there was no significant difference between the EC group and the EC+CM group (Fig. 7F).

\section{Discussion}

The mammalian heart is capable of regenerating to some extent; however, it is still unable to generate sufficient myocardial tissue to repair ischemic cardiomyopathy [20]. In 
recent years, a number of preclinical trials were conducted based on cell transplantation for myocardial repair, including the transplantation of foetal cardiomyocytes, skeletal myoblasts, smooth muscle cells, umbilical vein endothelial cells or bone marrow mononuclear cells [10]. Some of these tests yielded positive results, though the death of implanted cells, the presence of massive scar tissue and limited neovascularisation still hindered subsequent clinical applications [20-22].

In the current study, we found that endothelial cells regulated the reorganisation of cardiac myocytes in a $\beta 1$-integrin-signaling-dependent manner, with the two cell types synergistically promoting myocardial repair in an AMI mouse model, thereby suggesting a promising treatment for ischemic cardiomyopathy.

Our in vitro study showed that cardiomyocytes preferred migrating towards and staying beside endothelial cells, which was consistent with Narmoneva et al.'s observations in a 3-dimensional configuration [3]. Although the culture system used by Narmoneva et al. was optimised to mimic in vivo circumstances, the physical contact between the two cell populations limited the elucidation of the underlying mechanism. Herein, we used a transwell coculture system with ECs and CMs seeded separately on opposite sides of the filter to investigate CM transmigration and demonstrated that the chemotaxis of CMs towards ECs was contact independent and that $\beta 1$-integrin signalling played a pivotal role in the process. A similar result was obtained in a study of vascular smooth muscle cells (VSMCs) by Wang et al. [23], who showed that coculture with ECs enhanced VSMC adhesion and spreading via $\beta 1$-integrin signalling. Nevertheless, the present study is the first to demonstrate the $\beta 1$ integrin-dependent interaction of ECs and CMs.

Integrin is a transmembrane heterodimeric receptor, composed of $\alpha$ and $\beta$ subunits, that not only serves as a link between ECM ligands and cytoskeletal structures but also participates in bidirectional signal transduction associated with various biological processes, such as adhesion, spreading and migration [24]. Because integrins do not possess intrinsic kinase activity, cytoplasmic effectors must be triggered to transmit signals [25]. Focal adhesion kinase (FAK) is a primary integrin effector and can be activated by the integrin clustering $[25,26]$. Our results showed that coupled with the upregulation of $\beta 1$-integrin, ECs induced F-actin assembly and focal adhesion (FA) formation in cocultured CMs. Because $\beta 1$-integrin is essential for actin cytoskeleton assembly and because directed cell migration relies on actin-dependent lamellipodia formation at the leading edge of the cell $[19,23]$, these results indicated that $\beta 1$-integrin-dependent cytoskeletal reorganisation might be responsible for the EC-induced CM-reorganisation, though more work needs to be conducted to confirm this.

Recent studies have offered direct evidence for the pivotal role of $\beta 1$-integrin in stress responses in the heart [24, 27]. Using a mouse model featuring a cardiomyocyte-specific $\beta 1$-integrin deficiency, Li et al. showed that although cardiac function remained normal when $\beta 1$-integrin expression was reduced to $35 \%$, ventricular compaction was perturbed after hemodynamic stress, and this was accompanied by reduced FAK levels and alterations to multiple signalling pathways. In the meantime, when mice with cardiomyocyte-specific overexpression of $\beta 1 \mathrm{D}$ integrin were subjected to ischemia, the protective effect of $\beta 1 \mathrm{D}$ integrin on the myocardium during hypoxic injury was revealed by the substantially reduced infarct size compared with wild-type littermates [24]. Considering these previous findings, our present result that higher levels of $\beta 1$-integrin were observed in groups with better recovery from myocardial injury following AMI indicated that the therapeutic benefit elicited by ECs or ECs+CMs treatment might be explained by a $\beta 1$-integrin-related mechanism.

In the present study, we characterised the effect of ECs on CM reorganisation and cytoskeletal assembly and evaluated the therapeutic potential of combined EC-CM transplantation in an AMI mouse model. Our findings demonstrated the necessity for $\beta 1$-integrin in the interactions between CMs and ECs and presented a novel combined transplantation approach that might yield greater therapeutic promise for ischemic cardiomyopathy than the simple implantation of ECs or CMs alone. 


\section{Cellular Physiology Cell Physiol Biochem 2015;35:1808-1820 \begin{tabular}{l|l} 
and Biochemistry Published onlIne: IVIarch 26, 2015 & $\begin{array}{l}\text { C 2015 S. Karger AG, Basel } \\
\text { www.karger.com/cpb }\end{array}$ \\
\hline
\end{tabular}}

Zhang et al.: $\beta 1$-Integrin Mediated Endothelium-Cardiomyocyte Interaction

\section{Acknowledgements}

This work was supported by the National Natural Science Foundation of China (Grant No.31301211) and the Open Project Program of the Key Laboratory of Myocardial Ischemia of Harbin Medical University (Grant No.KF201317).

\section{Disclosure Statement}

The authors declare no competing interests

\section{References}

1 Ieda M, Tsuchihashi T, Ivey KN, Ross RS, Hong TT, Shaw RM, Srivastava D: Cardiac fibroblasts regulate myocardial proliferation through beta1 integrin signaling. Dev Cell 2009;16:233-244.

2 Howard CM, Baudino TA: Dynamic cell-cell and cell-ECM interactions in the heart. J Mol Cell Cardiol 2014;70:19-26.

- Narmoneva DA, Vukmirovic R, Davis ME, Kamm RD, Lee RT: Endothelial cells promote cardiac myocyte survival and spatial reorganization: implications for cardiac regeneration. Circulation 2004;110:962-968.

4 Winegrad S: Endothelial cell regulation of contractility of the heart. Annu Rev Physiol 1997;59:505-525.

-5 Brutsaert DL: Cardiac endothelial-myocardial signaling: its role in cardiac growth, contractile performance, and rhythmicity. Physiol Rev 2003;83:59-115.

6 Rhoads RP, Johnson RM, Rathbone CR, Liu X, Temm-Grove C: Satellite cell-mediated angiogenesis in vitro coincides with a functional hypoxia inducible factor pathway. Am J Physiol Cell Physiol 2009;296:C13211328.

7 Gorman JL, Liu ST, Slopack D, Shariati K, Hasanee A, Olenich S, Olfert IM, Haas TL: Angiotensin II evokes angiogenic signals within skeletal muscle through co-ordinated effects on skeletal myocytes and endothelial cells. PLoS One 2014;9:e85537.

8 Giordano FJ, Gerber HP, Williams SP, VanBruggen N, Bunting S, Ruiz-Lozano P, Gu Y, Nath AK, Huang Y, Hickey R, Dalton N, Peterson KL, Ross J Jr, Chien KR, Ferrara N: A cardiac myocyte vascular endothelial growth factor paracrine pathway is required to maintain cardiac function. Proc Natl Acad Sci U S A 2001;98:5780-5785.

9 Brzezinska AK, Merkus D, Chilian WM: Metabolic communication from cardiac myocytes to vascular endothelial cells. Am J Physiol Heart Circ Physiol 2005;288H2232-2237.

10 Schuh A, Liehn EA, Sasse A, Hristov M, Sobota R, Kelm M, Merx MW, Weber C: Transplantation of endothelial progenitor cells improves neovascularization and left ventricular function after myocardial infarction in a rat model. Basic Res Cardiol 2008; 103(1):69-77.

11 [11] Bouchentouf M, Paradis P, Forner KA, Cuerquis J, Boivin MN, Zheng J, Boulassel MR, Routy JP, Schiffrin EL, Galipeau J: Monocyte derivatives promote angiogenesis and myocyte survival in a model of myocardial infarction. Cell Transplant 2010;19:369-386.

12 Hristov M, Weber C: Endothelial progenitor cells: characterization, pathophysiology, and possible clinical relevance. J Cell Mol Med 2004;8:498-508.

13 Cheng Y, Hu R, Lv L, Ling L, Jiang S: Erythropoietin improves the efficiency of endothelial progenitor cell therapy after myocardial infarction in mice: effects on transplanted cell survival and autologous endothelial progenitor cell mobilization. J Surg Res 2012;176:e47-55.

14 Lim YC, Garcia-Cardena G, Allport JR, Zervoglos M, Connolly AJ, Gimbrone MA Jr, Luscinskas FW: Heterogeneity of endothelial cells from different organ sites in T-cell subset recruitment. Am J Pathol 2003;162:1591-1601.

15 Zhang Y, Li H, Lian Z, Li N: Myofibroblasts protect myoblasts from intrinsic apoptosis associated with differentiation via $\beta 1$ integrin-PI3K/Akt pathway. Dev Growth Differ 2010;52:725-733.

-16 Bilousova G, Jun du H, King KB, De Langhe S, Chick WS, Torchia EC, Chow KS, Klemm DJ, Roop DR, Majka SM: Osteoblasts derived from induced pluripotent stem cells form calcified structures in scaffolds both in vitro and in vivo. Stem Cells 2011;29:206-216. 


\section{Cellular Physiology Cell Physiol Biochem 2015;35:1808-1820

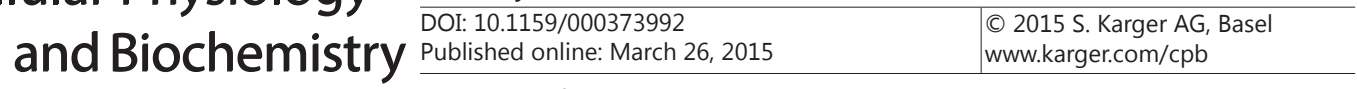 \\ Zhang et al.: $\beta 1$-Integrin Mediated Endothelium-Cardiomyocyte Interaction}

17 Xu L, Xu CJ, Lv HZ, Wang YX, Li Y, Lu PH: Long-term fate of allogeneic neural stem cells following transplantation into injured spinal cord. Stem Cell Rev and Rep 2010;6:121-136.

18 Li H, Zhang Y, Ning ZH, Deng XM, Lian ZX, Li N: Effect of selection for phagocytosis in dwarf chickens on immune and reproductive characters. Poult Sci 2008;87:41-49.

-19 Ballestrem C, Wehrle-Haller B, Hinz B, Imhof BA: Actin-dependent lamellipodia formation and microtubule-dependent tail retraction control-directed cell migration. Mol Biol Cell 2000;11:2999-3012.

20 Shantsila E, Watson T, Lip GY: Endothelial progenitor cells in cardiovascular disorders. J Am Coll Cardiol 2007;49:741-752.

-21 Anversa P, Leri A, Kajstura J: Cardiac regeneration. J Am Coll Cardiol 2006;47:1769-1776.

-22 Lam JT, Moretti A, Laugwitz KL: Multipotent progenitor cells in regenerative cardiovascular medicine. Pediatr Cardiol 2009;30:690-698.

23 Wang HQ, Bai L, Shen BR, Yan ZQ Jiang ZL: Coculture with endothelial cells enhances vascular smooth muscle cell adhesion and spreading via activation of beta1-integrin and phosphatidylinositol 3-kinase/Akt. Eur J Cell Biol 2007;86:51-62.

-24 Okada H, Lai NC, Kawaraguchi Y, Liao P, Copps J, Sugano Y, Okada-Maeda S, Banerjee I, Schilling JM, Gingras AR, Asfaw EK, Suarez J, Kang SM, Perkins GA, Au CG, Israeli-Rosenberg S, Manso AM, Liu Z, Milner DJ, Kaufman SJ, Patel HH, Roth DM, Hammond HK, Taylor SS, Dillmann WH, Goldhaber JI, Ross RS: Integrins protect cardiomyocytes from ischemia/reperfusion injury. J Clin Invest 2013;123:4294-4308. Yoshida Y, Togi K, Matsumae H, Nakashima Y, Kojima Y, Yamamoto H, Ono K, Nakamura T, Kita T,Tanaka M: CCN1 protects cardiac myocytes from oxidative stress via b1 integrin-Akt pathway. Biochem Biophys Res Commun 2007;355:611-618.

26 Ross RS, Borg TK: Integrins and the myocardium. Circ Res 2001;88:1112-1119.

-27 Li R, Wu Y, Manso AM, Gu Y, Liao P, Israeli S, Yajima T, Nguyen U, Huang MS, Dalton ND, Peterson KL, Ross RS: beta1 integrin gene excision in the adult murine cardiac myocyte causes defective mechanical and signaling responses. Am J Pathol 2012;180:952-962. 